\title{
ON THE RATIONAL FOUNDATIONS OF THE NON-EQUILIBRIUM THERMODYNAMICS OF ORDINARY FLUID MIXTURES
}

\author{
John L. BartelT* and Frederick H. Horne \\ Department of Chemistry, Michigan State University, \\ East Lansing, Michigan
}

\begin{abstract}
Despite the arbitrary and ambiguous character of certain fundamental parts of the usual theory of non-equilibrium thermodynamics of mixtures, it has been successful in describing a variety of experimental situations. The goals of the research partially reported in this paper have been (1) to strengthen the foundations of the usual, practical theory and (2) to extend its usefulness to experimental situations hitherto regarded as too complicated to analyse. The principles and methods of rational mechanics are used to deduce balance equations which take full account of the kinetic energy of each component and of the partial stress tensor of each component. Similar equations have been obtained previously by similar techniques. The new results reported here stem from a particular choice of independent variables; namely, for a $v$-component, non-reacting mixture of fluids, the independent variables are the $v$ component densities and their gradients, the temperature and its gradient, the $v-1$ independent diffusive velocities, and the $v$ symmetric and $v-1$ antisymmetric parts of the gradients of component velocity. For the special but very important case of an ordinary fluid mixture, whose constitutive relations are linear in the diffusion velocities and the independent gradients, the source term in the entropy balance equation is a bi-linear form in the diffusion velocities and the independent gradients. This bi-linear form for the entropy source term, which has eluded previous workers, has two immediate consequences: (1) Coupled with the second law, it leads to unambiguous conditions for equilibrium; namely, for equilibrium with respect to all processes in a $v$-component, non-reacting fluid mixture, it is necessary and sufficient that the following shall all vanish: the temperature gradient, the diffusion velocities, and the symmetric and antisymmetric parts of the gradients of component velocity. (2) It permits full comparison with the practical theory, wherein the entropy source plays a central role. It also opens new experimental avenues since it contains terms which arise from component kinetic energy and component stress tensor.
\end{abstract}

\section{INTRODUCTION}

The 'thermodynamics of irreversible processes" ${ }^{1}$ has enjoyed a history of practical success in describing a diversity of transport phenomena and a wide range of material behaviours. Despite its success and apparent completeness, the macroscopic theory as usually presented is deficient in several

\footnotetext{
* Now at Bell Telephone Laboratories, Inc., Murray Hill, New Jersey.
} 
respects, especially in its treatment of mixtures. The procedure for obtaining linear relations between fluxes and forces is arbitrary and ambiguous. 'Curie's theorem' is often used inconsistently, and rational account of the number of independent variables is seldom taken. The mechanics of stress and flow are adequately treated for pure fluids but not for mixtures of fluids; in the latter case only the composite fluid is considered. Terms arising from the kinetic energy of diffusion and the so-called inertial and viscous terms are always neglected in practical applications although explicit formulas are available. In short, the usual theory is really successful only for mechanical equilibrium, when pressure and velocity gradients vanish. In recent years Coleman and $\mathrm{Noll}^{2}$ and Coleman and Mizel ${ }^{3}$ have developed a new approach to thermodynamics built on a firm foundation in mechanics-precisely the area slighted in the conventional theory. In view of its mathematical origins it is not surprising that the new approach to thermodynamics is a model of rigour and that it remedies the deficiencies in mechanics of the conventional non-equilibrium theory, at least in the case of pure components (single materials).

Having great respect for both the practical success and microscopic relevance of the conventional theory and the rigour and completeness of the new approach, we present here a formulation of the thermodynamic theory of mixtures which is developed using Coleman's methods ${ }^{2,3}$, but which is aimed at reinforcing and supplementing the previous results. The approach here is quite similar to that of Müller ${ }^{4}$, and likewise has its origins in the work of Truesdell ${ }^{5}$. Müller, however, did not obtain the bi-linear form for the entropy production which is central to the earlier theory ${ }^{1}$. We obtain the bi-linear form by choosing expressions for the heat flux slightly different from, but no less general than, those of Müller. For brevity only the barest rudiments of the fundamental theory and only an outline of our methods are presented here. Full details may be found elsewhere ${ }^{6}$.

Generalized linear phenomenological equations are deduced rationally by application of the entropy inequality to general expressions, called constitutive relations, which relate dependent variables to a complete set of independent variables. In particular, we assume that the basic local mechanical and thermal variables energy, entropy, heat flux, entropy flux, stresses and interaction forces are determined by the basic independent variables temperature, temperature gradient, component densities, component density gradients, component velocities and component velocity gradients. The physical principles upon which the theory is constructed are: (i) balance of mass, energy, linear momentum, and angular momentum; (ii) equipresence, which means that an independent variable present in one constitutive relation is present in all unless its presence in a particular constitutive relation contradicts some other physical principle or any assumed symmetry of the material in question; (iii) material objectivity, which forbids descriptions which depend on the frame of reference of the observer, no matter how he moves; (iv) positive entropy production, which is nothing but the second law of thermodynamics, and which we call the entropy inequality. The procedure is to formulate the most general set of constitutive relations consistent with principles (i), (ii) and (iii) and with any other particular assumptions about the material in question. We then establish the necessary and sufficient 
conditions on the constitutive relations so that the entropy inequality holds identically. The resulting, reduced constitutive relations together with the basic dynamic equations comprise our complete set of macroscopic transport equations.

With Truesdell ${ }^{5}$, we assume that the mean motion of the fluid is determined solely by the motions of the components, and that the latter motions are determined by the usual stress properties plus interaction (exchange o momentum). For clarity, we restrict the treatment to binary mixtures there is no conceptual difficulty in extending it to any finite number or components. Since we wish to achieve the theory which corresponds most directly to the successful practical theor ${ }^{1}$, we further restrict the treatment to the special class of mixtures whose constitutive relations are linear in the temperature gradient, in the density gradients, and in objective combinations of the velocities and their gradients. A material of this special class will be called an ordinary binary fluid mixture. A conviction that this type of material accurately represents the binary fluid mixtures commonly encountered in the chemist's laboratory is the reason for the label 'ordinary'. The appropriateness of this label can be evaluated only by testing the ability of the constitutive relations to represent accurately the observed responses of the material to variations of independent variables. We neglect entirely the question of chemical reactions.

\section{THERMODYNAMIC PROCESS}

The generalization for mixtures of the definition of Coleman and $\mathrm{Noll}^{2}$ is: A process is a thermodynamic process for a $v$-component mixture if it can be described by the set of $(5 v+6)$ functions,

(i) the component densities $\rho_{\alpha}$; (ii) the component velocities $\mathbf{v}^{\alpha}$; (iii) the interaction forces $\mathbf{m}^{\alpha}$ acting on component $\alpha$ due to interaction with other components; (iv) the external body forces $\mathbf{b}^{\alpha}$ acting on component $\alpha$; (v) the partial stress tensors $\sigma^{\alpha}$; (vi) the specific internal energy $\varepsilon_{I}$; (vii) the temperature $T$; (viii) the radiative heat supply $r$ (radiation from the external world absorbed by the fluid); (ix) the heat flux q ; (x) the specific entropy $S$; and (xi) the entropy flux $\mathbf{f}$, which satisfy:

(a) the balance of mass for each component*,

where

$$
\dot{\rho}_{\alpha}+u_{k}^{\alpha}\left(\partial \rho_{\alpha} / \partial x_{k}\right)+\rho_{\alpha}\left(\partial v_{k}^{\alpha} / \partial x_{k}\right)=0
$$

$$
\rho=\sum_{\alpha=1}^{v} \rho_{\alpha}, \quad \rho \mathbf{v}=\sum_{\alpha=1}^{v} \rho_{\alpha} \mathbf{v}_{\alpha}, \quad \mathbf{u}_{\alpha}=\mathbf{v}_{\alpha}-\mathbf{v}, \quad \sum_{\alpha=1}^{v} \rho_{\alpha} \mathbf{u}_{\alpha}=0
$$

and where, for any function $g$,

$$
\dot{g}=(\partial g / \partial t)+v_{k}\left(\partial g / \partial x_{k}\right)
$$

(b) the balance of each vector component of linear momentum for each species,

$$
\rho_{\alpha} \dot{v}_{j}^{\alpha}-\left(\partial \sigma_{k j}^{\alpha} / \partial x_{k}\right)+\rho_{\alpha} u_{k}^{\alpha}\left(\partial v_{j}^{\alpha} / \partial x_{k}\right)=\rho m_{j}^{\alpha}+\rho_{\alpha} b_{j}^{\alpha}
$$

* A repeated Latin miniscule subscript on a vector or tensor indicates summation from 1 to 3 . 
(c) the balance of angular momentum for the mixture,

$$
\sum_{\alpha=1}^{v} \tau_{i j}^{\alpha}=0, \quad \tau_{i j}^{\alpha}=\frac{1}{2}\left(\sigma_{i j}^{\alpha}-\sigma_{j i}^{\alpha}\right)
$$

(d) the balance of energy

$$
\rho \dot{\varepsilon}_{I}+\left(\partial q_{k} / \partial x_{k}\right)=\rho r-\rho \sum_{\alpha=1}^{v} m_{k}^{\alpha} u_{k}^{\alpha}+\sum_{\alpha=1}^{v} \sigma_{j k}^{\alpha}\left(\partial v_{j} / \partial x_{k}\right)
$$

In order to specify a thermodynamic process it suffices to prescribe the $(4 v+5)$ functions $\rho_{\infty}, \mathbf{v}^{\alpha}, \mathbf{m}^{\alpha}, \sigma^{\alpha}, \varepsilon_{I}, T, \mathbf{q}, S$ and $\mathbf{f}$ since the remaining $(v+1)$ functions $\mathbf{b}^{\alpha}$ and $r$ are determined by (2.4) and (2.6). The specific internal energy $\varepsilon_{I}$ is the total specific energy $\varepsilon_{T}$ less the specific kinetic energy ${ }^{5,6}$,

$$
\varepsilon_{I}=\varepsilon_{T}-\sum_{\alpha=1}^{v} \frac{1}{2}\left(\rho_{\alpha} / \rho\right) v_{\alpha}^{2}
$$

The total stress tensor $\sigma$ is related to the component stress tensors by ${ }^{5,6}$

$$
\sigma=\sum_{\alpha=1}^{v}\left(\sigma^{\alpha}-\rho_{\alpha} \mathbf{u}^{\alpha} \mathbf{u}^{\alpha}\right)
$$

The component stress tensor here is identical with that of statistical mechanical theory?

\section{LINEAR CONSTITUTIVE RELATIONS FOR AN ORDINARY BINARY FLUID MIXTURE}

A material is defined by a constitutive assumption, which is a restriction on the processes admissible in a body consisting of the material. A mixture of viscoelastic materials susceptible of diffusion and heat conduction is completely determined by the values of the $(3 v+4)$ functions $A_{I}, S, \mathbf{q}, \mathbf{f}, \xi^{\alpha}$, $\tau^{\alpha}$ and $\mathbf{m}^{\alpha}$, where $A_{I}$ is the specific internal Helmholtz free energy, $A_{I}=\varepsilon_{I}$ $-T S$, and $\xi^{\alpha}$ is the symmetric part of $\sigma^{\alpha}$,

$$
\xi_{i j}^{\alpha}=\frac{1}{2}\left(\sigma_{i j}^{\alpha}+\sigma_{j i}^{\alpha}\right)
$$

Müller ${ }^{4}$ showed that the entropy flux need not be specifically related a priori to the heat flux, but instead may be given by a constitutive relation whose form is determined by the basic principles in the same way as other constitutive relations.

The subsequent exposition is restricted to the special material called an ordinary binary fluid mixture. For such mixtures the constitutive relations are ordinary functions of $\rho_{1}, \rho_{2}$ and $T$ and are linear functions of $\left(\partial \rho_{1} / \partial x_{k}\right)$, $\left(\partial \rho_{2} / \partial x_{k}\right),\left(\partial T / \partial x_{k}\right), u_{k}^{1}, \mathrm{~d}_{j k}^{1}, \mathrm{~d}_{j k}^{2}$ and $\omega_{j k}^{1}$, where

$$
\mathrm{d}_{j k}^{\alpha}=\frac{1}{2}\left[\left(\partial v_{j}^{\alpha} / \partial x_{k}\right)+\left(\partial v_{k}^{\alpha} / \partial x_{j}\right)\right]
$$

and

$$
\omega_{j k}^{1}=\frac{1}{2}\left\{\left[\left(\partial u_{j}^{1} / \partial x_{k}\right)-\left(\partial u_{k}^{1} / \partial x_{j}\right)\right]+\rho^{-1} \sum_{\alpha=1}^{2}\left[u_{j}^{\alpha}\left(\partial \rho_{\alpha} / \partial x_{k}\right)-u_{k}^{\alpha}\left(\partial \rho_{\alpha} / \partial x_{j}\right)\right]\right\}
$$


The constitutive relations for an ordinary binary fluid mixture are ${ }^{6}$ :

$$
\begin{aligned}
& A_{I}=A_{I}\left(\rho_{1}, \rho_{2}, T\right), S=S\left(\rho_{1}, \rho_{2}, T\right) \\
& q_{j}=-C_{q T}\left(\partial T / \partial x_{j}\right)-C_{q 1}\left(\partial \rho_{1} / \partial x_{j}\right)-C_{q 2}\left(\partial \rho_{2} / \partial x_{j}\right)-C_{q u} u_{j}^{1} \\
& m_{j}^{1}=-C_{m T}\left(\partial T / \partial x_{j}\right)-C_{m 1}\left(\partial \rho_{1} / \partial x_{j}\right)-C_{m 2}\left(\partial \rho_{2} / \partial x_{j}\right)-C_{m u} u_{j}^{1} \\
& f_{j}=-C_{f T}\left(\partial T / \partial x_{j}\right)-C_{f 1}\left(\partial \rho_{1} / \partial x_{j}\right)-C_{f 2}\left(\partial \rho_{2} / \partial x_{j}\right)+K u_{j}+\left(q_{j} / T\right) \\
& \tau_{i j}^{1}=-\kappa \omega_{i j}^{1} \\
& \xi_{i j}^{\alpha}=-\pi_{\alpha} \delta_{i j}+\sum_{\beta=1}^{2} \phi_{\alpha \beta} \mathrm{d}_{k k}^{\beta} \delta_{i j}+\sum_{\beta=1}^{v} 2 \eta_{\alpha \beta}\left(\mathrm{d}_{i j}^{\beta}-\frac{1}{3} \mathrm{~d}_{k k}^{\beta} \delta_{i j}\right)
\end{aligned}
$$

where $d_{k k}$ is the trace of the tensor $d^{\beta}$.

These constitutive relations have been deduced $^{6}$ from a starting set of constitutive relations of the form

$$
R=R\left[\rho_{1}, \rho_{2}, T,\left(\partial \rho_{1} / \partial x_{j}\right),\left(\partial \rho_{2} / \partial x_{j}\right),\left(\partial T / \partial x_{j}\right), u_{j}^{1}, u_{j}^{2}, \mathrm{~d}_{i j}^{1}, \mathrm{~d}_{i j}^{2}, \omega_{i j}^{1}\right]
$$

Compared with general theory of the conventional sort ${ }^{1}$, the most important feature of (3.5) is the explicit enumeration of independent variables. Compared with the theory of Müller ${ }^{4}$, the most important feature of (3.5) is the particular choice of independent variables. Our choice permits us to obtain many more consequences from the entropy inequality than does Müller's choice. Our choice leads to unambiguous definitions of various types of equilibrium, and to a bi-linear form for entropy production.

The thermodynamic processes possible for a particular material must of course depend on the material. Since it is the constitutive relations which define a material, we define an admissible thermodynamic process as a thermodynamic process which is compatible with the constitutive relations for the material in question. In particular, an admissible thermodynamic process for an ordinary binary fluid mixture must be compatible with (3.4). Application, in Section IV, of the entropy inequality provides restrictions on the phenomenological coefficients of (3.4) but affects neither the choice of independent variables nor the forms of the constitutive relations.

\section{THE ENTROPY INEQUALITY AND ITS CONSEQUENCES}

Entropy and entropy flux are related by the balance equation

$$
\rho \dot{S}=-\left(\partial f_{k} / \partial x_{k}\right)+\rho \Phi+(\rho r / T)
$$

where $\Phi$ is the rate of specific entropy production not due to external radiation sources. Specification of an admissible thermodynamic process determines $\Phi$ through the constitutive relations for $S$ and $\mathbf{f}$. The second law of thermodynamics in this context is the Postulate ${ }^{2}$ : For every admissible thermodynamic process the inequality

$$
\Phi \geqslant 0
$$

must hold at all times and at every point in the material. That is, the entropy production is non-negative, identically in the independent variables. In order 
to conserve space, we omit the details ${ }^{6}$ of the exploitation of (4.2). Repeated application of (4.2) and full utilization of the independence of the variables of (3.4) yield a host of consequences, which we now list :

\section{(a) Equilibrium theorem}

An ordinary binary fluid mixture is at equilibrium if and only if each of the independent variables $\left(\partial T / \partial x_{k}\right), u_{k}^{1}, \mathrm{~d}_{j k}^{1}, \mathrm{~d}_{j k}^{2}$ and $\omega_{j k}^{1}$ is zero. Vanishing of the density gradients or of the barycentric velocity is not required for equilibrium.

\section{(b) Entropy equations}

For an ordinary binary fluid mixture, a deductive consequence of the principles of rational mechanics is

$$
S=-\left(\partial A_{I} / \partial T\right) \rho_{1}, \rho_{2}
$$

which is a familiar identity in thermostatics. Each of the first three coefficients of $(3.4)_{4}$ vanishes, and the entropy flux becomes

$$
\mathbf{f}=K u^{1}+(\mathbf{q} / T)
$$

The final bi-linear form for the entropy production is

$$
\begin{gathered}
\rho T \Phi=\left(C_{q T} / T\right)\left(\partial T / \partial x_{k}\right)\left(\partial T / \partial x_{k}\right)+\Theta_{T} u_{k}^{1}\left(\partial T / \partial x_{k}\right)+\left(\rho^{2} / \rho_{2}\right) C_{m u} u_{k}^{1} u_{k}^{1} \\
+\sum_{\alpha=1}^{2} \sum_{\beta=1}^{2} \phi_{\alpha \beta} \mathrm{d}_{i i}^{\alpha} \mathrm{d}_{j j}^{\beta}+ \\
+\left(\rho / \rho_{1}^{2} \rho_{2}\right) \kappa \omega_{i j}^{1} \omega_{i j}^{1} \\
+\sum_{\alpha=1}^{2} \sum_{\beta=1}^{2} 2 \eta_{\alpha \beta}\left(\mathrm{d}_{i j}^{\alpha}-\frac{1}{3} \mathrm{~d}_{k k}^{\alpha} \delta_{i j}\right)\left(\mathrm{d}_{i j}^{\beta}-\frac{1}{3} \mathrm{~d}_{k k}^{\beta} \delta_{i j}\right)
\end{gathered}
$$

where

$$
\Theta_{T}=T(\partial K / \partial T)_{\rho_{1}, \rho_{2}}+\left(C_{q u} / T\right)+\left(\rho^{2} / \rho_{2}\right) C_{m T}
$$

\section{(c) Viscosity coefficients}

For an ordinary binary fluid mixture there appear to be nine (not two) viscosity coefficients, restricted only by the conditions:

$$
\begin{gathered}
\kappa \geqslant 0, \\
\phi_{11} \geqslant 0, \phi_{22} \geqslant 0,\left[\phi_{11} \phi_{22}-\frac{1}{4}\left(\phi_{12}+\phi_{21}\right)^{2}\right] \geqslant 0, \\
\eta_{11} \geqslant 0, \eta_{22} \geqslant 0,\left[\eta_{11} \eta_{22}-\frac{1}{4}\left(\eta_{12}+\eta_{21}\right)^{2}\right] \geqslant 0 .
\end{gathered}
$$

\section{(d) Heat flux equation}

For an ordinary binary fluid mixture, $C_{q 1}$ and $C_{q 2}$ vanish, and $(3.4)_{2}$ becomes

$$
q_{j}=-C_{q T}\left(\partial T / \partial x_{j}\right)-C_{q u} u_{j}^{1}
$$

This is precisely the heat of transport form of the heat flux equation in the practical theory ${ }^{1 d}$, with $C_{q T}$ the thermal conductivity coefficient, $\rho_{i} \mathbf{u}^{1}$ the diffusion flux $\mathbf{j}^{1}$ and $\left(-C_{q u} / \rho_{1}\right)$ the heat of transport $Q_{1} \star$. 


\section{(e) Diffusion flux equation}

Rearrangement of $(3.4)_{3}$ yields

$$
\begin{aligned}
\rho_{1} u_{j}^{1}=-\left(\rho_{1} C_{m T} / C_{m u}\right) & \left(\partial T / \partial x_{j}\right)-\left(\rho_{1} C_{m 1} / C_{m u}\right)\left(\partial \rho_{1} / \partial x_{j}\right) \\
& -\left(\rho_{1} C_{m 2} / C_{m u}\right)\left(\partial \rho_{2} / \partial x_{j}\right)-\left(\rho_{1} / C_{m u}\right) m_{j}^{1}
\end{aligned}
$$

For an ordinary binary fluid mixture, (4.2) requires:

$$
\begin{aligned}
& C_{m u} \geqslant 0,\left[\left(\rho^{2} / \rho_{2}\right) C_{q T} C_{m u}-\frac{1}{4} \Theta_{T}^{2}\right] \geqslant 0 \\
& C_{m 1}=\left(\rho_{2} / \rho^{3}\right) T K-\left(\rho_{2} / \rho^{2}\right)\left(\partial K / \partial \rho_{1}\right)_{T, \rho_{2}}-\left(\rho_{2} / \rho\right)\left(\partial A_{I} / \partial \rho_{1}\right)_{T, \rho_{2}} \\
& C_{m 2}=-\left(\rho_{1} / \rho^{3}\right) T K-\left(\rho_{2} / \rho^{2}\right) T\left(\partial K / \partial \rho_{2}\right)_{T, \rho_{1}}+\left(\rho_{1} / \rho\right)\left(\partial A_{I} / \partial \rho_{2}\right)_{T, \rho_{1}}
\end{aligned}
$$

Further reduction of these equations must await identification of the coefficient $K$ which occurs explicitly not only in $(3.4)_{4},(4.4),(4.6)$ and (4.10), but also in two further consequences of (4.2) for an ordinary binary fluid mixture; namely, the $\pi_{\alpha}$ of (3.4) ${ }_{6}$ must be given by:

$$
\begin{aligned}
& \pi_{1}=\rho \rho_{1}\left(\partial A_{I} / \partial \rho_{1}\right)_{T, \rho_{2}}+\left(\rho_{2} / \rho\right) T K \\
& \pi_{2}=\rho \rho_{2}\left(\partial A_{I} / \partial \rho_{2}\right)_{T, \rho_{1}}-\left(\rho_{2} / \rho\right) T K
\end{aligned}
$$

The $\pi_{\alpha}$ may be called partial pressures since

$$
\pi_{1}+\pi_{2}=\rho\left[\rho_{1}\left(\partial A_{1} / \partial \rho_{1}\right)_{T, \rho_{2}}+\rho_{2}\left(\partial A_{I} / \partial \rho_{2}\right)_{T, \rho_{1}}\right]=P
$$

\section{ACKNOWLEDGEMENT}

Support of this research by the National Science Foundation is gratefully acknowledged. Moreover, Predoctoral Fellowships to JLB from the Public Health Service and the Lubrizol Foundation are gratefully acknowledged.

\section{REFERENCES}

1 (a) J. Meixner and H. G. Reik, in Handbuch der Physik, S. Flügge, (Ed). Springer: Berlin (1959). Vol. III/2; (b) J. G. Kirkwood and B. Crawford Jr, J. Phys. Chem. 56, 1048 (1952); (c) S. R. de Groot and P. Mazur, Non-Equilibrium Thermodynamics. North Holland Publ. Co.: Amsterdam (1962); (d) D. D. Fitts, Non-equilibrium Thermodynamics. McGraw-Hill: New York (1962); (e) R. Haase, Thermodynamics of Irreversible Processes. Addison-Wesley: Reading, Mass. (1969).

${ }^{2}$ B. D. Coleman and W. Noll, Arch. Ration. Mech. Anal. 13, 167 (1963).

3 B. D. Coleman and V. J. Mizel, (a) Arch. Ration. Mech. Anal. 13, 245 (1963); (b) J. Chem. Phys. 40, 1116(1964).

4 I. Müller, Arch. Ration. Mech. Anal. 26, 118 (1967): (b) Arch. Ration. Mech. Anal. 28, 1 (1968). 5 (a) C. Truesdell, R.C. Acad. Lincei, (8), 22, 33 (1957), reprinted in The Rational Mechanics of Materials, pp 293-305. C. Truesdell (Ed). Gordon and Breach: New York (1965); (b) C. Truesdell and R. A. Toupin, in Handbuch der Physik, Vol. III/1, S. Flügge (Ed). Springer: Berlin (1960); (c) C. Truesdell and W. Noll, in Handbuch der Physik, Vol. III/3. S. Flügge (Ed.). Springer : Berlin (1965).

6 J. L. Bartelt, Ph.D. Thesis. Michigan State University (1968).

7 R. J. Bearman and J. G. Kirkwood, J. Chem. Phys. 28, 136 (1958). 